\title{
BMJ
}

\section{Effect of statin treatment on short term mortality after pneumonia episode: cohort study}

\author{
Ian Douglas, lecturer in epidemiology, Stephen Evans, professor of pharmacoepidemiology, Liam Smeeth, \\ professor of clinical epidemiology
}

Faculty of Epidemiology and Population Health, London School of Hygiene and Tropical Medicine, London WC1E 7HT, UK

Correspondence to: I Douglas ian.douglas@lshtm.ac.uk

Cite this as: $B M J$ 2011;342:d1642 doi:10.1136/bmj.d1642

\section{ABSTRACT}

Objective To determine whether statins protect against all cause mortality after a diagnosis of pneumonia.

Design Cohort study using propensity score based method to control for differences between people prescribed and not prescribed statins.

Setting United Kingdom Health Improvement Network database, which contains electronic primary care medical records of more than six million patients.

Participants Every patient starting a statin between 1995 and 2006 (129 288) matched with up to five non-statin users ( $n=600241) ; 9073$ patients had a recorded diagnosis of pneumonia, of whom 1398 were using a statin.

Main outcome measure All cause mortality within six months of diagnosis of pneumonia.

Results Among users and non-users of statins with comparable propensity scores, 95/942 users and 686/ 3615 non-users died on the day that pneumonia was diagnosed. In the following six month period, 109/847 statin users died compared with 578/2927 non-users, giving an adjusted hazard ratio of 0.67 (0.49 to 0.91 ). If these observed benefits translated into clinical practice, 15 patients would need to be treated with a statin for six months after pneumonia to prevent one death.

Conclusions Compared with people who were not taking statins, the risk of dying in the six month period after pneumonia was substantially lower among people who were already established on long term statin treatment when the pneumonia occurred. Whether some or all of this protective effect would be obtained if statin treatment begins when a patient first develops pneumonia is not known. However, given that statins are cheap, safe, and well tolerated, a clinical trial in which people with pneumonia are randomised to a short period of statin treatment is warranted.

\section{INTRODUCTION}

Statins inhibit hydroxymethyl glutaryl coenzyme A reductase, which means that they lower blood cholesterol concentrations and hence reduce the risk of ischaemic cardiac events. ${ }^{12}$ Over the past decade, much has been claimed about the possible wide ranging effects of statins. ${ }^{3}$ Although the pharmacology of statins theoretically supports a wide range of actions, many benefits seen in observational studies are possibly due to indication bias and evidence for them has been inconsistent. ${ }^{45}$

A recent systematic review highlighted a possible role for statins in the treatment and prevention of infections. ${ }^{6}$ The results of nine studies of statin treatment for a range of mostly bacterial infections were pooled, and a protective effect was found against various outcomes including sepsis and mortality, with an odds ratio of 0.55 (95\% confidence interval 0.36 to $0.83)$. This would suggest that patients receiving statins have less severe outcomes after infection.

We previously reported findings from a large cohort study comparing users and non-users of statins for a range of outcomes. ${ }^{4} \mathrm{We}$ validated our study design by comparing the effects on mortality and cardiovascular events seen in our cohort compared with the effects seen in large randomised trials. The cardiovascular effect sizes were remarkably similar, suggesting that we had succeeded in overcoming the selection bias and confounding that is a common problem in observational studies of effects of drugs. In our previous study, we reported a possible reduction in the incidence of pneumonia among people receiving statins (hazard ratio $0.84 ; 99 \%$ confidence interval 0.74 to $0.95)$. In practice, statins would be unlikely to be given widely to prevent infections because of the very large numbers of people who would need long term treatment to prevent incident disease. However, statins may have a more useful role during acute infections, with the aim of lessening severity. We therefore investigated the effect of statins on mortality after an episode of pneumonia.

\section{METHODS}

The Health Improvement Network

The Health Improvement Network (THIN) is a database of computerised medical records of general practices in the United Kingdom. It includes diagnostic and prescribing information, as well as information on various lifestyle characteristics for more than six million patients from more than 300 general practices. Patients are representative of the general UK population; rates of pregnancy, consultation, drug prescribing, and death are similar to national figures, ${ }^{7}$ and the database 
has been validated for pharmacoepidemiological research. ${ }^{8}$

\section{Participants}

The methods used to assemble the cohort for this study have been described in detail elsewhere. ${ }^{4}$ Briefly, all patients aged over 40 years registered in THIN between January 1995 and December 2006 were eligible for inclusion. We included in the study all patients who received their first prescription for a statin on or after 1 January 1995 and had at least 12 months' previous continuous registration in THIN. We termed the date of first statin prescription the index date. We matched up to five non-statin users to each statin user on sex and age within 5 years. Non-users had to be registered at the same practice as their matched user on the index date, with at least 12 months' previous continuous registration. Non-users had to have no record of a prescription for a statin before the index date but could be prescribed a statin later. This approach avoids selecting a biased control group who were not at risk of being prescribed a statin. We required both users and non-users of statins to have had some form of contact with their practice within six months before or after the index date to ensure that all participants were active patients.

\section{Analysis}

\section{Propensity score}

A propensity score is a measure of the probability that a patient will be prescribed a certain drug, in this case a statin, based on the distribution of perceived risk factors for use among participants who have used and not used statins. Propensity scores are useful in observational studies, as they allow the researcher to determine whether groups of users and non-users are truly comparable, and the score can then be used as a way of handling confounding. ${ }^{9}$ We calculated propensity scores for all patients, regardless of whether they subsequently developed pneumonia, by using conditional logistic regression with use of statin at the index date as the outcome. Factors included in the model were those likely to influence the decision to prescribe a statin and included observation time in THIN; body mass index; socioeconomic status; consultation rate; prescribing rate; smoking status; drinking habits; diabetes; coronary heart disease; cerebrovascular disease; peripheral vascular disease; other atheroma; other circulatory disease; dementia; cancer; atrial fibrillation; heart failure; recent hepatic disease; recent renal disease; thyroid disease; hyperlipidaemia; hypertension; recent use of hormone replacement therapy, antipsychotics, antidepressants, steroids (oral or inhaled), fibrates, or cytochrome P450 3A4 inhibitors; and any previous use of non-statin or fibrate lipid lowering drugs, nitrates, aspirin, $\beta$ blockers, calcium channel blockers, potassium channel activators, diuretics, positive inotropes, anticoagulants, antihypertensives, or other cardiovascular drugs. As a sensitivity analysis, we also calculated propensity scores restricted to the reduced cohort of patients who developed pneumonia.
Propensity scores can be used to adjust for confounding in several ways. ${ }^{910}$ Patients with similar scores can be matched individually, or the score can be adjusted for in regression analysis. Alternatively, analyses can be restricted to specific strata within the distribution of the score. Whichever approach is used, patients should be included in comparative analyses only if their score falls within the range common to both users and nonusers, thus avoiding comparisons between patients with too many differences other than the exposure of interest. In addition, patients with scores in the extreme upper or lower tail of the distribution of scores should be excluded, as patients may be treated contrary to their score for important reasons, often missing to the researcher but related to health outcomes. ${ }^{10}$ Their inclusion can therefore lead to biased estimates. We examined the distribution of scores to ensure that only patients with scores in common regions of the distribution were included, and, as a minimum, excluded those with scores in the upper and lower $5 \%$ of the distribution from analyses. We also did a sensitivity analysis without this exclusion. From past experience, we anticipated that the distribution of scores would not allow efficient matching of users to non-users and would require adjustment for the score in regression analyses. We used likelihood ratio testing to determine whether the score should be treated as a continuous or categorical variable. We constructed 20 categories of $5 \%$ each for this purpose.

\section{Exposure}

Two exposures were relevant for this study. Firstly, all patients included in the primary analysis had to have had an episode of incident pneumonia after the index date, as identified by patients' medical records. For patients with more than one episode of pneumonia, we included only the first episode in the analysis. Secondly, we determined use of statin at the date when pneumonia was recorded. We considered patients who had received a prescription for a statin in the 60 day period before pneumonia to be users; we considered all others to be non-users.

\section{Outcome}

The outcome of interest in this study was all cause mortality within six months of the incident episode of pneumonia. For patients with at least one episode of pneumonia after the index date, we ascertained death from patients' medical records and defined the date of death as the earliest of any records indicating that death had occurred.

\section{Modelling strategy}

Firstly, we separated people who died on the day that pneumonia was diagnosed from those who died later. This is because pneumonia recorded on the date of death is likely to represent a contributing factor to the death rather than an incident diagnosis. Patterns of recording of cause of death are likely to be influenced by a patient's known underlying comorbidities, so differences in the likelihood of pneumonia being cited as 
Table 1 |Characteristics of study population at index date. Values are numbers (percentages)

\begin{tabular}{|c|c|c|c|c|}
\hline \multirow[b]{2}{*}{ Characteristics } & \multicolumn{2}{|c|}{ All patients } & \multicolumn{2}{|c|}{$\begin{array}{l}\text { Patients with a post-index date episode } \\
\text { of pneumonia }\end{array}$} \\
\hline & $\begin{array}{l}\text { Non-statin users } \\
(n=600241)\end{array}$ & $\begin{array}{l}\text { Statin users } \\
(n=129288)\end{array}$ & $\begin{array}{l}\text { Non-statin users } \\
\quad(n=7675)\end{array}$ & $\begin{array}{c}\text { Statin users } \\
(n=1398)\end{array}$ \\
\hline \multicolumn{5}{|l|}{ Age (years): } \\
\hline $40-45$ & $29221(4.9)$ & $5613(4.3)$ & $110(1.4)$ & $27(1.9)$ \\
\hline $45-50$ & $45230(7.5)$ & $9258(7.2)$ & $222(2.9)$ & $25(1.8)$ \\
\hline $50-55$ & $71140(11.9)$ & 14548 (11.3) & $390(5.1)$ & $102(7.3)$ \\
\hline $55-60$ & 92756 (15.5) & $19383(15.0)$ & $604(7.9)$ & $137(9.8)$ \\
\hline $60-65$ & $96292(16.0)$ & $20959(16.2)$ & $929(12.1)$ & $189(13.5)$ \\
\hline $65-70$ & 90895 (15.1) & $20748(16.1)$ & $1231(16.0)$ & $238(17.0)$ \\
\hline $70-75$ & 74385 (12.4) & 17527 (13.6) & $1378(18.0)$ & $299(21.4)$ \\
\hline $75-80$ & $53995(9.0)$ & $12228(9.5)$ & 1336 (17.4) & $225(16.1)$ \\
\hline $80-85$ & $31119(5.2)$ & $6230(4.8)$ & $873(11.4)$ & $91(6.5)$ \\
\hline $85-90$ & $11980(2.0)$ & $2274(1.8)$ & $463(6.0)$ & $50(3.6)$ \\
\hline$\geq 90$ & $3228(0.5)$ & $520(0.4)$ & 139 (1.8) & $15(1.1)$ \\
\hline Male sex & $299158(49.8)$ & $65517(50.7)$ & 4058 (52.9) & $780(55.8)$ \\
\hline \multicolumn{5}{|l|}{ Smoking status: } \\
\hline Non-smoker & 201062 (33.5) & 31578 (24.4) & $2009(26.2)$ & $280(20.0)$ \\
\hline Ex-smoker & $152273(25.4)$ & $46018(35.6)$ & $1972(25.7)$ & $516(36.9)$ \\
\hline Current smoker & $221779(37.0)$ & $50383(39.0)$ & $3139(40.9)$ & $592(42.4)$ \\
\hline Unknown & $25127(4.2)$ & $1309(1.0)$ & $555(7.2)$ & $10(0.7)$ \\
\hline \multicolumn{5}{|l|}{ Body mass index: } \\
\hline$<20$ & $24098(4.0)$ & $2619(2.0)$ & $673(8.8)$ & $48(3.4)$ \\
\hline $20-25$ & $175760(29.3)$ & $33971(26.3)$ & $2284(29.8)$ & $370(26.5)$ \\
\hline 125 & 308695 (51.4) & $83919(64.9)$ & $3080(40.1)$ & $875(62.6)$ \\
\hline Missing & $91688(15.3)$ & $8779(6.8)$ & $1638(21.3)$ & $105(7.5)$ \\
\hline \multicolumn{5}{|l|}{ Alcohol consumption: } \\
\hline Non-drinker & $80273(13.4)$ & $13236(10.2)$ & $1266(16.5)$ & $186(13.3)$ \\
\hline Ex-drinker & $4428(0.7)$ & $1104(0.9)$ & $82(1.1)$ & $17(1.2)$ \\
\hline Drinker, quantity unknown & $16795(2.8)$ & $5704(4.4)$ & $231(3.0)$ & $68(4.9)$ \\
\hline$<2$ units/day & $348425(58.1)$ & $76325(59.0)$ & $3870(50.4)$ & $797(57.0)$ \\
\hline 3-6 units/day & $64833(10.8)$ & 20525 (15.9) & $754(9.8)$ & $188(13.5)$ \\
\hline$>6$ units/day & $8751(1.5)$ & $4285(3.3)$ & $174(2.3)$ & $58(4.2)$ \\
\hline Status unknown & 76736 (12.8) & $8109(6.3)$ & $1298(16.9)$ & $84(6.0)$ \\
\hline \multicolumn{5}{|l|}{ Comorbidities: } \\
\hline Atherosclerosis & $68358(11.4)$ & $63607(49.2)$ & 1965 (25.6) & $986(70.5)$ \\
\hline Heart failure & $12946(2.2)$ & $6551(5.1)$ & $711(9.3)$ & $218(15.6)$ \\
\hline Hypertension & $173101(28.8)$ & $68538(53.0)$ & 2567 (33.5) & $753(53.9)$ \\
\hline Dementia & $3932(0.7)$ & $329(0.3)$ & $226(2.9)$ & $10(0.7)$ \\
\hline Cancer & $32535(5.4)$ & $6246(4.8)$ & $742(9.7)$ & $93(6.7)$ \\
\hline Diabetes & $53955(9.0)$ & 35699 (27.6) & $953(12.4)$ & $450(32.2)$ \\
\hline \multicolumn{5}{|l|}{ Drugs: } \\
\hline Antipsychotics & $10360(1.7)$ & 1552 (1.2) & $394(5.1)$ & $22(1.6)$ \\
\hline Antidepressants & $56947(9.5)$ & $15165(11.7)$ & $1188(15.5)$ & $222(15.9)$ \\
\hline Aspirin & 68776 (11.5) & $62728(48.5)$ & 1785 (23.3) & $871(62.3)$ \\
\hline
\end{tabular}

a cause of death between statin users and non-users are likely. For this reason, we did no formal analyses on data from patients who died on the date when pneumonia was recorded. For patients who died later or not at all, the recording of pneumonia is more likely to be truly incident; we did formal survival analysis for these patients.

We used Cox regression to model mortality in the six month period after an episode of pneumonia, excluding all patients who died on the day of diagnosis of pneumonia. Right censoring occurred at the earliest of six months after recording of pneumonia, death, or the end of the observation period (transfer out of practice or last data collection date).

We initially adjusted analyses for age in five year bands and sex. Fully adjusted analyses accounted for age, sex, propensity score, and year of index date. We produced a Kaplan-Meir survival curve to investigate how the risk of death changed over the six months after pneumonia, excluding deaths on the day of diagnosis. 
Table 2 | Distribution of propensity scores*

\begin{tabular}{|c|c|c|c|c|}
\hline \multirow{2}{*}{$\begin{array}{l}\text { Propensity } \\
\text { score in } \\
20 \text { groups* }\end{array}$} & \multicolumn{2}{|c|}{ Non-statin users } & \multicolumn{2}{|c|}{ Statin users } \\
\hline & No (\%) & Deaths (\%) & No (\%) & Deaths (\%) \\
\hline 1 & $576(7.5)$ & $86(15)$ & $1(0.07)$ & $1(100)$ \\
\hline 2 & $576(7.5)$ & $91(16)$ & $2(0.14)$ & 0 \\
\hline 3 & $496(6.5)$ & $81(16)$ & $2(0.14)$ & 0 \\
\hline 4 & $470(6.1)$ & 80 (17) & $2(0.14)$ & 0 \\
\hline 5 & $498(6.5)$ & $69(14)$ & $1(0.07)$ & 0 \\
\hline 6 & $451(5.9)$ & $63(14)$ & $5(0.36)$ & $1(20)$ \\
\hline 7 & $425(5.5)$ & $52(12)$ & $6(0.43)$ & 0 \\
\hline 8 & 449 (5.9) & $66(15)$ & $4(0.29)$ & 0 \\
\hline 9 & 374 (4.9) & $55(15)$ & $8(0.6)$ & $2(25)$ \\
\hline 10 & $404(5.3)$ & 73 (18) & $13(0.9)$ & $2(15)$ \\
\hline 11 & $390(5.1)$ & $56(14)$ & $17(1.2)$ & $4(24)$ \\
\hline 12 & $368(4.8)$ & $55(15)$ & $22(1.6)$ & $3(14)$ \\
\hline 13 & $333(4.3)$ & $51(15)$ & 24 (1.7) & $2(8)$ \\
\hline 14 & $325(4.2)$ & $56(17)$ & $39(2.8)$ & $2(5)$ \\
\hline 15 & $311(4.1)$ & $53(17)$ & $36(2.6)$ & $2(6)$ \\
\hline 16 & $325(4.2)$ & 62 (19) & $83(5.9)$ & $15(18)$ \\
\hline 17 & $365(4.8)$ & $61(17)$ & $145(10.4)$ & $14(10)$ \\
\hline 18 & $250(3.3)$ & $32(13)$ & 239 (17.1) & 31 (13) \\
\hline 19 & $170(2.2)$ & 24 (14) & 316 (22.6) & $32(10)$ \\
\hline 20 & 119 (1.6) & $9(8)$ & $433(31.0)$ & 48 (11) \\
\hline
\end{tabular}

*1=lowest likelihood of receiving statin; 20=highest likelihood of receiving statin; based on factors included in propensity score.

To illustrate the observed effect size, we calculated the number needed to treat (NNT) by using the equation $\mathrm{NNT}=1 /$ (risk among non-statin users with pneumonia -risk among statin users with pneumonia). For comparison, we also used the same approach to model the effect of statin use on all cause mortality in all patients, regardless of pneumonia status, after the index date. We used Stata 11 for all analyses.

\section{RESULTS}

We matched 129288 people who had received a statin to 600241 people not prescribed a statin (the complete cohort). Smeeth et al give a detailed breakdown of patients' characteristics among statin users and nonusers. ${ }^{4}$ Table 1 shows background information in the entire cohort and in the patients with a diagnosis of pneumonia. Among these, 9121 people had an episode of incident pneumonia after the index date (date of first statin prescription in statin users). In 137 cases, death was recorded before pneumonia. Of these, 109 had a period of 35 days or shorter between recorded death and pneumonia, indicating that pneumonia may have been recorded as a possible cause of death. In these cases, we brought the date of pneumonia forward to the date of death. The remaining 28 cases had a longer time lag between recorded death and pneumonia, and we excluded them from further consideration owing to uncertainty about the accuracy of dating of events. For a further 23 patients, the recorded end of observation occurred before the record of pneumonia. In these patients, pneumonia was possibly a retrospective recording. Where the period between end of observation and pneumonia was 35 days or shorter, we changed the date of pneumonia to the end of observation $(n=4)$. For longer periods, we excluded patients from further analyses owing to uncertainty about accuracy of dating $(n=19)$. This left 9073 patients, of whom $1398(15 \%)$ were receiving a statin at the time of diagnosis of pneumonia and 7675 were not receiving a statin. Table 1 also gives descriptive characteristics of the patients with pneumonia. Of note, 4869/9073 (54\%) people with pneumonia were aged 70 years or older compared with $213486 / 729529(29 \%)$ in the complete cohort. Patients with pneumonia were also more likely to have a history of atherosclerosis $(2957 / 9073(33 \%) v$ $131965 / 729529(18 \%))$ or heart failure $(929 / 9073$ $(10 \%)$ v $19497 / 729529(3 \%))$.

Very few patients who used statins had propensity scores in the lower eight $5 \%$ propensity score bands, as shown in table 2; further analysis therefore excluded patients with scores in these bands, in addition to the 20th band, to ensure that we drew comparisons only between patients with scores in the range seen in both users and non-users of statins. After this restriction, the cohort consisted of 942 statin users and 3615 nonusers, of whom 204 users and 1264 non-users died within six months of the date pneumonia was recorded. Among these patients, $95(10 \%)$ users and $686(19 \%)$ non-users died on the day pneumonia was recorded. A further 109 (13\%) of the 847 remaining users and 578/2927 (20\%) non-users died later during the six month follow-up period and were entered in the survival analysis (table 3).

The rate ratio for mortality over six months, excluding deaths on the day of diagnosis of pneumonia, was 0.62 (99\% confidence interval 0.47 to 0.81 ), adjusted for age and sex and comparing statin users with nonusers. The fully adjusted rate ratio was 0.67 (0.49 to 0.87 ). The figure shows the crude Kaplan-Meier survival estimates for statin users and non-users, excluding deaths on the date of diagnosis of pneumonia. A sensitivity analysis using propensity scores calculated only among patients who developed pneumonia gave a

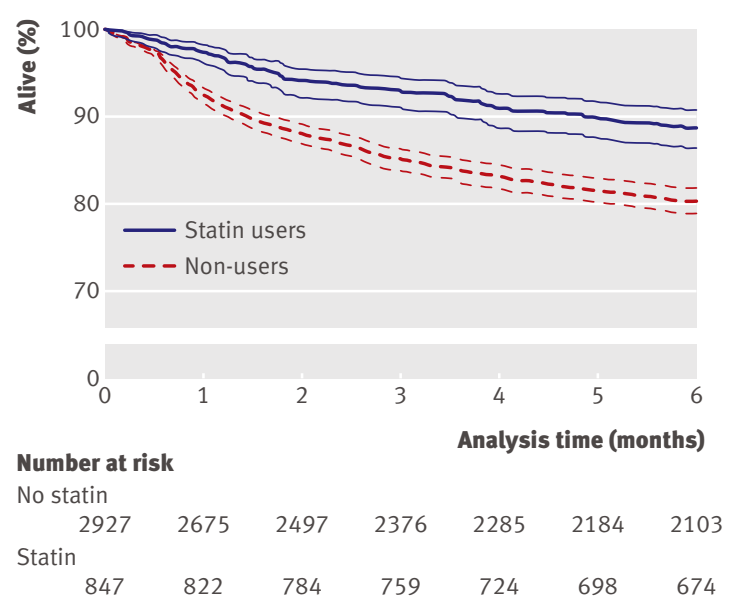

Kaplan-Meier survival estimates showing crude mortality estimates (with $95 \%$ confidence intervals) in six months after episode of pneumonia in statin users and non-users, excluding deaths on day of diagnosis 
Table 3 |Association between use of statin and mortality

\begin{tabular}{|c|c|c|c|c|}
\hline Outcome & No of patients & No (\%) deaths & $\begin{array}{l}\text { Age and sex adjusted } \\
\text { effect estimate: hazard } \\
\text { ratio }(99 \% \mathrm{Cl})\end{array}$ & $\begin{array}{c}\text { Fully adjusted effect } \\
\text { estimate: hazard ratio } \\
(99 \% \mathrm{Cl})\end{array}$ \\
\hline \multicolumn{5}{|c|}{ Mortality on day of pneumonia diagnosis } \\
\hline Did not use statin & 3615 & $686(19)$ & & \\
\hline Used statin & 942 & $95(10)$ & & \\
\hline \multicolumn{5}{|c|}{ Mortality within 6 months of pneumonia, excluding deaths on day of pneumonia* } \\
\hline Did not use statin & 2927 & $578(20)$ & - & - \\
\hline Used statin & 847 & $109(13)$ & $0.62(0.47$ to 0.81$)$ & 0.67 (0.49 to 0.91$)$ \\
\hline \multicolumn{5}{|c|}{ All mortality after index date (regardless of pneumonia status) ${ }^{\star}$} \\
\hline Did not use statin & 308730 & $32445(11)$ & - & - \\
\hline Used statin & 51397 & $4718(9)$ & 0.95 (0.91 to 0.99$)$ & 0.82 (0.79 to 0.86$)$ \\
\hline
\end{tabular}

*Analysis restricted to patients with comparable propensity scores.

fully adjusted rate ratio of 0.65 (0.49 to 0.87$)$. By contrast, a comparison of patients who stopped taking a statin with those who never started taking one gave a fully adjusted rate ratio of 0.82 ( 0.50 to 1.36$)$ for all further deaths in the next six months. This could reflect a loss of protective effect due to stopping statin treatment, although uncontrolled differences between groups may also have contributed to the null effect seen. A sensitivity analysis including patients with a score in the 20th $5 \%$ score band yielded a similar result to the primary analysis, with a hazard ratio of $0.72(0.54$ to 0.96$)$. A sensitivity analysis stratified on propensity score did not suggest that the result was restricted to any particular region of the score distribution (see web appendix).

Considering mortality in the complete cohort, 4718/ $51397(9 \%)$ patients who were prescribed a statin at the index date died during follow-up compared with $32445 / 308730(11 \%)$ not prescribed a statin, after restriction of analysis to patients within propensity score bands 9 to 19 . The rate ratio for mortality, adjusted for age and sex, comparing statin users with non-users was 0.95 (0.91 to 0.99$)$. The fully adjusted rate ratio was 0.82 (0.79 to 0.86 ).

\section{Number needed to treat}

If the estimated effect was seen in a randomised trial, the number needed to treat to prevent death within six months of an episode of pneumonia episode would be $1 /((578 / 2927)-(109 / 847))=15$.

\section{DISCUSSION}

Compared with people who were not taking statins, the risk of dying in the six month period after pneumonia was substantially lower among people who were already established on long term statin treatment when the pneumonia occurred, with a hazard ratio of 0.67 (99\% confidence interval 0.49 to 0.91 ). The effect seen seems to be greater than the effect of statins on all cause mortality regardless of pneumonia status, which was $0.82(0.79$ to 0.86$)$ in this cohort.

\section{Strengths and limitations of study}

A major strength of this study is the large cohort size; nearly 5000 episodes of pneumonia were included in the main analysis, and around 1000 of these were in patients prescribed statins. In observational studies of effects of drugs, a major problem is ensuring that confounding by indication has been dealt with so that the result is likely to represent an effect of the drug rather than fundamental differences between treated and untreated people. We were able to adjust for a large number of confounders by using propensity scores; this approach can successfully account for much confounding by indication. By examining the distribution of scores separately in the statin users and non-users, we were able to restrict our main analysis to people with a similar likelihood of receiving a statin. This is an advantage of propensity score methods compared with more traditional multivariate analysis, in which the comparability of users and non-users can be more difficult to determine.

As with all observational studies, we cannot be certain that the effects we observed are causally related to the use of statins. Although the possibility of residual confounding by indication and other biases can never be ruled out, we believe that when making observations among statin users and non-users, vascular events are likely to be subject to the most substantial confounding by indication. We have previously shown a protective effect against myocardial infarction and stroke in this dataset, similar to the effect detected in clinical trials. ${ }^{4}$ Moreover, for confounding by indication to explain these results people at higher risk of severe pneumonia would have to be preferentially not prescribed statins. Although people with immunocompromising illness may be treated less often for reduction of lipids, this could be expected to lead to a similar bias for mortality after influenza as well as pneumonia. We previously reported no benefit of statin treatment for complications or mortality after influenza; a point estimate of 0.84 suggested no extra benefit compared with the generally expected benefit of statins against mortality. ${ }^{4}$ This suggests that our approach may have successfully eliminated much confounding by indication, although obviously this is not absolutely certain.

For this study, we chose to consider deaths on the day of diagnosis of pneumonia separately from deaths that occurred later, as in these cases pneumonia may have been recorded only because it was given as a cause of death. We were concerned that pneumonia could be cited as a cause of death in a way that systematically differs between statin users and non-users. Recording of pneumonia as a cause of death has been problematic in the UK until very recently, when specific guidance was issued for certification of death. ${ }^{11}$

A degree of misclassification of exposure and outcome may have occurred in this study. General practitioners may not have recorded some episodes of pneumonia (for example, if a patient was admitted to hospital and died shortly after, without pneumonia being noted on the death certificate). Assuming that such misclassification was similar for statin users and non-users, this would be expected to lead to a reduction in power only. Alternatively, some patients could 


\section{WHAT IS ALREADY KNOWN ON THIS TOPIC}

A recent systematic review highlighted a possible role for statins in the treatment and prevention of infections

Pneumonia is a common infection that often leads to serious morbidity or death

The widescale use of statins as a preventive measure is likely to be unfeasible, but their use in treating pneumonia could be a simple and inexpensive adjunct to current strategies

\section{WHAT THIS STUDY ADDS}

Established statin use seems to be associated with a substantial reduction in the risk of death shortly after an episode of pneumonia

Whether starting a statin at the time pneumonia is diagnosed carries similar benefit is not known

A randomised trial is warranted to establish whether these findings translate into clinical practice

have been incorrectly diagnosed as having pneumonia, which would result in a bias towards the null. Bias could also be introduced if pneumonia was recorded differently among statin users and nonusers. Unfortunately, we could not ascertain the completeness and validity of recording of pneumonia in this study.

Recording of mortality in THIN is comparable with UK-wide rates, ${ }^{7}$ but some deaths may not have been recorded. Again, this is unlikely to have differed according to statin use and would therefore be expected to bias any result towards the null. Finally, use of statins is of vital importance; the primary analysis allocated statin use as determined by prescription rather than consumption. Some patients do not take their drugs as prescribed, so, at the time pneumonia occurred, a small proportion of patients may be misclassified as statin users. However, more than $99 \%$ of patients taking statins had received two or more prescriptions for statins, and repeat prescribing is likely to represent genuine drug use in most cases. Misclassification of statin use is also unlikely to have differed according to mortality status, so we would expect the effect to be a bias towards the null. The observed protective effect of statins seemed to be much diminished or absent when we compared patients who stopped their statin with those who never started. Caution is needed in the interpretation of this result, however, as people who stop their statin may differ fundamentally from those who continue treatment, in ways that are directly related to underlying health.

\section{Comparison with other studies}

Observational studies in other populations have looked at the effect of statins in the treatment of pneumonia but have had limited power. Our results seem to be largely in keeping with those reported elsewhere. Four studies identified a protective effect when comparing statin users with non-users. Almog et al reported an odds ratio of 0.07 (0.01 to 0.51 ) for severe sepsis in patients with bacterial infections $(50 \%$ of which were pneumonia), although the enormous magnitude of effect detected in this study suggests possible residual confounding. ${ }^{12}$ Frost et al found a hazard ratio of 0.61 (0.41 to 0.92 ) for mortality due to influenza/pneumonia, although what proportion of these cases were pneumonia is unclear. ${ }^{13}$ Mortensen et al reported an odds ratio of 0.48 ( 0.36 to 0.64 ) for mortality 30 days after pneumonia. ${ }^{14}$ Myles et al found a hazard ratio of 0.33 (0.19 to 0.58 ) for all cause mortality within 30 days of pneumonia. ${ }^{15}$ Of note, this study also used data from THIN but included only 12 fatal cases among statin users, compared with 216 in our study. In contrast, Majumdar et al found an odds ratio of 1.1 (0.76 to 1.6) for all cause mortality after admission to hospital for community acquired pneumonia. ${ }^{16}$ This study seems to be well conducted, and the investigators were able to account for many underlying factors likely to differ between statin users and non-users. The reasons for such differences in results are not clear.

\section{Implications of findings}

Our study was able to measure only the effect of statins as given before pneumonia developed; in many instances statin treatment had been ongoing for a long period of time. For these findings to be of direct clinical relevance, whether a short course of statin treatment started at the time of diagnosis of suspected pneumonia improves the chances of survival would need to be determined. A growing body of evidence shows that statins exert rapid effects; endothelial function and arterial flow improve within 24 hours of starting a statin, ${ }^{17}$ and inflammatory markers such as $\mathrm{C}$ reactive protein are reduced with three days. ${ }^{18}$

Our study was not designed to assess the mechanism of any observed protective effect. However, statins are known to affect the cell signalling pathways involved in immune and anti-inflammatory responses, which could lead to a reduction in severity of infection in some cases. ${ }^{19}$

Statins are safe,$^{20}$ cheap, and an easy intervention in terms of delivery. Given the potential low number needed to treat to prevent a death suggested by this study, we believe that a strong case exists for randomised trials of statins in people with serious infection to determine if a simple and practical intervention at the point of diagnosis of pneumonia has a beneficial effect. $^{2122}$ A trial of around 3000 people randomised to a statin or placebo at the time of diagnosis of pneumonia would be needed to determine whether a short period of statin treatment could reduce mortality by $20 \%$ among people with suspected pneumonia. Such a trial would be able to look in detail at specific causes of death, which may further elucidate a possible mechanism for any beneficial effect.

We thank EPIC for supplying the data and all the general practices that participate in the THIN database.

Contributors: ID and LS were responsible for the conception and design of the study. ID had principal responsibility for analysis, drafting and revising the manuscript, and final approval. LS and SE contributed to the analysis and the drafting, revision, and final approval of the manuscript. All authors were responsible for interpretation of the data. ID is the guarantor. Funding: During this work, ID was funded by grants from the Wellcome Trust and the Medical Research Council paid to the London School of Hygiene and Tropical Medicine. LS was funded by a grant from the 
Wellcome Trust. Sponsors played no role in the study design; the collection, analysis, and interpretation of data; the writing of the article; or the decision to submit it for publication

Competing interests: All authors have completed the Unified Competing Interest form at www.icmje.org/coi_disclosure.pdf (available on request from the corresponding author) and declare that (1) they have no relationships with companies that might have an interest in the submitted work in the previous three years; (2) their spouses, partners, or children have no financial relationships that may be relevant to the submitted work; and (3) they have no non-financial interests that may be relevant to the submitted work.

Ethical approval: The South Thames Multi-centre Research Ethics Committee and the London School of Hygiene and Tropical Medicine Ethics Committee approved the study.

Data sharing: No additional data available.

1 Heart Protection Study Collaborative Group. MRC/BHF Heart Protection Study of cholesterol lowering with simvastatin in 20,536 high-risk individuals: a randomised placebo-controlled trial. Lancet 2002;360:7-22.

2 Nissen SE, Tuzcu EM, Schoenhagen P, Crowe T, Sasiela W], Tsai ], et al. Statin therapy, LDL cholesterol, C-reactive protein, and coronary artery disease. N Engl J Med 2005;352:29-38.

3 Liao JK. Effects of statins on 3-hydroxy-3-methylglutaryl coenzyme a reductase inhibition beyond low-density lipoprotein cholesterol. $A m$ J Cardiol 2005;96:24-33F.

4 Smeeth L, Douglas I, Hall AJ, Hubbard R, Evans S. Effect of statins on a wide range of health outcomes: a cohort study validated by comparison with randomized trials. Br J Clin Pharmacol 2009;67:99-109.

5 Lodi S, Evans SJW, Egger P, Carpenter J. Is there an anti-inflammatory effect of statins in rheumatoid arthritis? Analysis of a large routinely collected claims database. Br J Clin Pharmacol 2009;69:85-94.

6 Tleyjeh IM, Kashour T, Hakim FA, Zimmerman VA, Erwin PJ, Sutton AJ, et al. Statins for the prevention and treatment of infections: $a$ systematic review and meta-analysis. Arch Intern Med 2009;169:1658-67.

7 Bourke A, Dattani H, Robinson M. Feasibility study and methodology to create a quality-evaluated database of primary care data. Inform Prim Care 2004;12:171-7.

8 Lewis JD, Schinnar R, Bilker WB, Wang X, Strom BL. Validation studies of the health improvement network (THIN) database for pharmacoepidemiology research. Pharmacoepidemiol Drug Saf 2007;16:1195-2002.
9 Sturmer T, Joshi M, Glynn RJ, Avorn J, Rothman KJ, Schneeweiss S. A review of the application of propensity score methods yielded increasing use, advantages in specific settings, but not substantially different estimates compared with conventional multivariable methods. J Clin Epidemiol 2006;59:437-47.

10 Sturmer, Rothman KJ, Avorn J, Glynn RJ. Treatment effects in the presence of unmeasured confounding: dealing with observations in the tails of the propensity score distribution-a simulation study. Am J Epidemiol 2010;172:843-54.

11 Office for National Statistics. Guidance for doctors completing medical certificates of cause of death in England and Wales: from the Office for National Statistics' Death Certification Advisory Group. ONS, 2008.

12 Almog Y, Shefer A, Novack V, Maimon N, Barski L, Eizinger M, et al. Prior statin therapy is associated with a decreased rate of severe sepsis. Circulation 2004;110:880-5.

13 Frost FJ, Petersen H, Tollestrup K, Skipper B. Influenza and COPD mortality protection as pleiotropic, dose-dependent effects of statins. Chest 2007;131:1006-12.

14 Mortensen EM, Restrepo MI, Anzueto A, Pugh J. The effect of prio statin use on 30-day mortality for patients hospitalized with community-acquired pneumonia. Respir Res 2005;6:82.

15 Myles PR, Hubbard RB, Gibson JE, Pogson Z, Smith CJ, McKeever TM. The impact of statins, ACE inhibitors and gastric acid suppressants on pneumonia mortality in a UK general practice population cohort. Pharmacoepidemiol Drug Saf 2009;18:697-703.

16 Majumdar SR, McAlister FA, Eurich DT, Padwal RS, Marrie TJ. Statin and outcomes in patients admitted to hospital with community acquired pneumonia: population based prospective cohort study. BMJ 2006;333:999.

17 Laufs U, Wassmann S, Hilgers S, Ribaudo N, Bohm M, Nickenig G. Rapid effects on vascular function after initiation and withdrawal of atorvastatin in healthy, normocholesterolemic men. Am J Cardiol 2001;88:1306-7.

18 Li JJ, Fang CH, Qian HY, Hu WL. Time course of rapid C-reactive protein reduction by pravastatin in patients with stable angina. Angiology 2006;57:1-7.

19 Kwak B, Mulhaupt F, Myit S, Mach F. Statins as a newly recognised type of immunomodulator. Nat Med 2000:6;1399-402.

20 Armitage J. The safety of statins in clinical practice. Lancet 2007;370:1781-90.

21 Hackam DG, Mamdani, M, Li P, Redelmeir DA. Statins and sepsis in patients with cardiovascular disease: a population based cohort study. Lancet 2006;367:413-8.

22 Terblanche M, Almog Y, Rosenson RS, Smith TS, Hackam DG. Statins: panacea for sepsis? Lancet Infect Dis 2006;6:242-8.

Accepted: 21 February 2011 